\title{
ESTUDO DO EFEITO DO TRATAMENTO SUPERFICIAL POR MOAGEM SOBRE AS PROPRIEDADES DAS PARTÍCULAS DE UMA LIGA DE HIDRETO METÁLICO
}

\author{
Elki Cristina Souza e Edson A. Ticianelli* \\ Instituto de Química de São Carlos, Universidade de São Paulo, CP 780, 13560-970 São Carlos - SP
}

Recebido em 17/12/04; aceito em 22/7/05; publicado na web em 3/2/06

\begin{abstract}
EFFECT OF A MILLING SURFACE TREATMENT ON THE PROPERTIES OF METAL HYDRIDE ALLOY PARTICLES. This work reports results of studies on the electrochemical and structural properties of a Ti/Zr-based metal hydride alloy covered by $\mathrm{Ni}$ and $\mathrm{LaNi}_{4,7} \mathrm{Sn}_{0,3}$ powder additives by ball milling. The effect of this treatment is investigated for the activation time, hydrogen storage capacity and equilibrium pressure, cycling stability and the hydration/dehydration kinetics. Charge and discharge cycles show a significant decrease of the activation time due to an increase of the active area caused by the milling treatment, independent of the additive. However, other results have evidenced little effect of the milling surface treatment on the charge storage capacity, hydrogen equilibrium pressure, and hydration/dehydration kinetics, for both the $\mathrm{Ni}$ and $\mathrm{LaNi}_{4,7} \mathrm{Sn}_{0,3}$ covered materials.
\end{abstract}

Keywords: electrode materials; hydrogen storage materials; ball milling.

\section{INTRODUÇÃO}

As famílias de ligas de hidreto metálico conhecidas atualmente apresentam composições $\mathrm{AB}, \mathrm{AB}_{2}, \mathrm{~A}_{2} \mathrm{~B}$ e $\mathrm{AB}_{5}$, onde nas classes $\mathrm{AB}$ e $\mathrm{A}_{2} \mathrm{~B}$, a parte $\mathrm{A}$ é geralmente baseada em $\mathrm{Mg}$ e $\mathrm{B}$ em Ni${ }^{1-3}$. Já nas $\mathrm{AB}_{5}$ e $\mathrm{AB}_{2}$ mais estudadas, $\mathrm{A}$ representa um metal de terras raras para a liga $\mathrm{AB}_{5}$ e um metal de transição de alto número atômico para a liga $\mathrm{AB}_{2}$; em ambos os casos, $\mathrm{B}$ pode incluir vários metais de transição de baixo ou alto números atômicos ${ }^{4-6}$. As ligas $\mathrm{AB}, \mathrm{A}_{2} \mathrm{~B}$ e $\mathrm{AB}_{2}$ tipicamente têm uma capacidade de armazenamento de carga superior que as ligas $\mathrm{AB}_{5}$, em muitos casos maiores que $500 \mathrm{mAh} / \mathrm{g}$, comparada com $300 \mathrm{mAh} / \mathrm{g}$ para a liga $\mathrm{AB}_{5}^{7,8}$.

As ligas tipo $\mathrm{AB}_{2}$ mais estudadas podem ser compostas por Ti$\mathrm{Zr}-\mathrm{V}-\mathrm{Ni}$-Cr e/ou outros elementos, onde $\mathrm{Ti}$ e $\mathrm{Zr}$ representam o componente A. Estas ligas podem ser também formadas à base de $\mathrm{Mg}$ e Ni, porém os materiais obtidos têm-se apresentado bastante instáveis. Ligas armazenadoras de hidrogênio dos tipos $\mathrm{AB}, \mathrm{A}_{2} \mathrm{~B}$ e $\mathrm{AB}_{2}$ baseadas em $\mathrm{Mg}$ possuem maior capacidade de absorção de hidrogênio quando comparadas com os demais $\operatorname{casos}^{1-3,9-11}$. No entanto, por um longo período, tem-se pensado que estas ligas não seriam apropriadas para uso como eletrodos negativos em baterias de Ni-MH, devido à cinética de hidretação/desidretação muito baixa em temperatura ambiente e à alta corrosão em eletrólito alcalino ${ }^{9,10}$. Atualmente, alguns trabalhos de pesquisas têm mostrado que estes são materiais promissores, pois permitem aumentar consideravelmente a capacidade do eletrodo negativo.

Recentemente, tem-se sugerido que um processo de moagem usando moinho de bolas de pós de materiais formados por mistura de ligas armazenadoras de hidrogênio pode levar a melhoras nas propriedades de ativação, na capacidade de armazenamento de carga e na estabilidade, em relação aos materiais sem este tratamento. Este método tem sido empregado a fim de mudar várias propriedades dos materiais pois permite uma mistura dos componentes a nível atômico, decorrente de um intenso trabalho mecânico que pode levar à formação de misturas e ligas nanocristalinas, misturas e ligas amorfas, microestruturas homogêneas, dispersão de fases insolúveis, recobrimentos de partículas, etc ${ }^{12}$. Em algumas ligas

*e-mail: edsont@iqsc.usp.br armazenadoras de hidrogênio, a formação de nanocristais ou estruturas amorfas por moagem resulta em uma dramática mudança nas propriedades de absorção e dessorção de hidrogênio, especialmente pela melhora da cinética de hidretação/desidretação ${ }^{9-15}$. Uma melhora do desempenho das ligas por moagem também tem sido obtida através de modificações superficiais das partículas da liga ativa, promovidas pela incorporação de metais puros, como Pd e $\mathrm{Ni}$, e compósitos, como $\mathrm{Co}_{3} \mathrm{Mo}$ e $\mathrm{LaNi}_{5}{ }^{13}$.

Outros trabalhos ${ }^{12,14}$ relatam o tratamento de ligas que são inicialmente processadas separadamente por fusão e depois moídas juntas em diferentes proporções em moinho de bolas, utilizando baixa energia e tempos curtos de moagem (de 15 a $40 \mathrm{~min}$ ). Componentes minoritários (aditivos) misturados a diversas ligas convencionais e não-convencionais armazenadoras de hidrogênio tipo $A B_{2}$ têm sido empregados. Os compósitos formados têm sido caracterizados por DRX (difração de raios X), sendo usualmente constatado que seus componentes individuais mantém-se distintos e que os aditivos estão na forma de finas camadas envolvendo as partículas da liga base. Os materiais têm sido caracterizados quanto ao comportamento eletroquímico, sendo observado que as principais vantagens dos eletrodos fabricados com esses compósitos são ativação inicial mais rápida; aumento significativo na cinética dos processos eletródicos; diminuição no sobrepotencial do processo de carga; melhora do comportamento cíclico e, em alguns casos, um aumento considerável na capacidade de armazenamento de carga em relação ao componente majoritário não tratado.

Neste trabalho foi feito um estudo das propriedades estruturais e do comportamento eletroquímico frente à reação de oxidação de hidrogênio, de eletrodos formados por pó de liga de hidreto metálico $\mathrm{Zr}_{0.9} \mathrm{Ti}_{0,1} \mathrm{Ni}_{1,04} \mathrm{Mn}_{0,64} \mathrm{~V}_{0,46}$ tratada por moagem em moinho de bolas na presença de pó de $\mathrm{Ni}$ ou da liga $\mathrm{LaNi}_{4,7} \mathrm{Sn}_{0,3}$ (tipo $\mathrm{AB}_{5}$ ). Foram investigados os efeitos destes tratamentos sobre o tempo de ativação, a capacidade de armazenamento de hidrogênio, a estabilidade dos eletrodos de hidreto metálico formados com estes materiais e a cinética de hidretação/desidretação.

\section{PARTE EXPERIMENTAL}

A liga base $\mathrm{Zr}_{0,9} \mathrm{Ti}_{0,1} \mathrm{Ni}_{1,04} \mathrm{Mn}_{0,64} \mathrm{~V}_{0,46}$ foi preparada através da 
fusão dos elementos metálicos de alta pureza $(99,9 \%)$ em forno a arco sob atmosfera de argônio ultra-seco (5,0 analítico, White Martins). O procedimento envolveu a repetição da fusão por várias vezes com reposicionamento do corpo de prova para garantir a homogeneidade de composição. Após a fusão, as ligas preparadas foram acondicionadas em ampolas de quartzo onde se fez vácuo, sendo, em seguida, submetidas a um tratamento térmico à temperatura de $950{ }^{\circ} \mathrm{C}$ por $72 \mathrm{~h}$. Após a fusão, as ligas foram pulverizadas mecanicamente com auxílio de um almofariz e pistilo, até as partículas possuírem diâmetro médio da ordem de $40 \mu \mathrm{m}$. O procedimento para recobrimento superficial das partículas com os aditivos metálicos consistiu na mistura do pó da liga estudada com $10 \%$ em peso de $\mathrm{LaNi}_{4,7} \mathrm{Sn}_{0,3}$ pulverizada $(\sim 30 \mu \mathrm{m})$, ou pó de $\mathrm{Ni}(\sim 5 \mu \mathrm{m})$, que foram acomodadas na cuba de moagem. Utilizou-se bolas de aço em uma proporção em massa 5/1 bola/material, sendo todo o processo efetuado por 40 min sob atmosfera de argônio.

Para se verificar a composição das ligas estudadas, utilizou-se a técnica de energia dispersiva de raios $\mathrm{X}$ (EDX) realizada em um equipamento LEO 440 com filamento de tungstênio, acoplado a um detector de energia dispersiva de raios X. Para se caracterizar a estrutura cristalina, obtiveram-se os difratogramas das amostras pulverizadas, com e sem os tratamentos por moagem, utilizandose um difratômetro universal de raios X modelo URD6 - Zeiss Zena. Utilizou-se a radiação $\mathrm{CuK} \alpha(\lambda=1,54 \AA)$ e um intervalo de $2 \theta$ varrido de $20^{\circ}$ a $80^{\circ}$, a uma velocidade de $2^{\circ}$ por min.

Os eletrodos de hidreto metálico foram preparados prensandose uma mistura formada por $0,05 \mathrm{~g}$ do pó da liga com $0,05 \mathrm{~g}$ de carbono de alta área superficial (Vulcan XC-72R) e 33\% em peso do agente ligante politetrafluoretileno (PTFE, Teflon T30, E. I. DuPont), em ambos os lados de uma tela de níquel com área de aproximadamente $2,0 \mathrm{~cm}^{2}$. Todos os experimentos eletroquímicos foram realizados em uma solução eletrolítica de $\mathrm{KOH}$ (Mallinckrodt) à uma concentração de $6,0 \mathrm{~mol} \mathrm{~L}^{-1}$; como eletrodo auxiliar foi utilizada uma tela de $\mathrm{Pt}$ e como eletrodo de referência $\mathrm{Hg} / \mathrm{HgO}, \mathrm{KOH}$ $6,0 \mathrm{~mol} \mathrm{~L}^{-1}$.

Nos testes de carga/descarga, o eletrodo negativo foi carregado a $5 \mathrm{~mA}$ por $6 \mathrm{~h}$ e descarregado em uma mesma corrente, adotando-se um potencial de corte de $-0,7 \mathrm{~V}$ vs. $\mathrm{Hg} / \mathrm{HgO}$. Os diagramas de impedância eletroquímica foram obtidos no potencial de circuito aberto, com eletrodos em diferentes estados de carga e temperaturas $\left(0,12,26\right.$ e $\left.38^{\circ} \mathrm{C}\right)$, utilizando-se um analisador de impedância Autolab (modelo PGSTAT 30), no intervalo de frequiência de $10 \mathrm{kHz}$ a 1 $\mathrm{mHz}$, a uma amplitude AC de $5 \mathrm{mV}$, com o objetivo de se obter parâmetros como a resistência de transferência de carga e a energia de ativação da reação de oxidação do hidrogênio.
Para as medidas da variação da pressão de equilíbrio de hidrogênio nas ligas em função do estado de carga, partiu-se do eletrodo ativado e $100 \%$ carregado, anotando-se o potencial a circuito aberto (considerado quando o potencial não variava mais de $1 \mathrm{mV} \mathrm{du}-$ rante $10 \mathrm{~min}$ ). $\mathrm{O}$ processo foi repetido, descarregando o eletrodo gradualmente, até que chegasse ao estado completamente descarregado. A pressão de equilíbrio de hidrogênio foi calculada através do potencial de equilíbrio $\left(\mathrm{E}_{M H}^{e q}\right)$, utilizando-se a Equação de Nernst ${ }^{16,17}$.

\section{RESULTADOS E DISCUSSÃO}

Os pós das ligas $\mathrm{AB}_{2}\left(\mathrm{Zr}_{0,9} \mathrm{Ti}_{0,1} \mathrm{Ni}_{1,04} \mathrm{Mn}_{0,64} \mathrm{~V}_{0,46}\right)$ não tratadas e tratadas com $10 \%$ dos aditivos metálicos foram caracterizados para se obter as proporções atômicas dos elementos que constituem cada material. A Tabela 1 mostra os resultados das análises por energia dispersiva de raios $\mathrm{X}$ (EDX). Os valores obtidos para as proporções atômicas dos elementos constituintes das ligas, dentro da margem de erro da técnica utilizada (cerca de 10\%), permitiram concluir que as composições de todas as ligas são inteiramente consistentes com as proporções atômicas definidas nominalmente (porcentagens teóricas). Para todas as ligas $\mathrm{AB}_{2}$ que foram submetidas ao tratamento por moagem, aparece uma certa porcentagem de $\mathrm{Fe}$ (cerca de 2,5\% em átomos) e, em menor proporção, de $\mathrm{Cr}$ (cerca de 0,5\%). Estes elementos fazem parte da constituição da cuba e das bolas de aço utilizadas no moinho e contaminam as amostras durante a moagem.

A Figura 1 mostra o difratograma de raios $X$ do pó da liga $A_{2}$ sem tratamento por moagem. Os picos identificados pelos índices de Miller incluídos na Figura 1 indicam a presença de uma fase hexagonal tipo $\mathrm{C} 14$ (estrutura tipo- $\mathrm{MgZn}_{2}$ ), que é característica destes materiais ${ }^{18}$. $\mathrm{O}$ difratograma não exibe picos de difração relativos aos metais constituintes da liga, fato que indica uma boa homogeneidade composicional. Os parâmetros de rede e o correspondente volume de cela unitária calculados a partir dos difratogramas resultaram em $a=4,9837 \AA$, $c=8,1969 \AA$ e V = $176,32 \AA^{3}$, sendo concordantes com os valores apresentados em outros trabalhos ${ }^{19}$ para uma liga com composição nominal semelhante.

Padrões de difração de raios $\mathrm{X}$ para as ligas com e sem aditivos depois do processamento por moagem são apresentados na Figura 2. Estas curvas de difração mostram uma diminuição na intensidade e um leve alargamento dos picos de difração de raios $\mathrm{X}$ para os materiais depois de submetidos ao tratamento por moagem. Este fato se deve exclusivamente a uma diminuição do tamanho dos

Tabela 1. Porcentagens atômicas calculadas e obtidas experimentalmente para as ligas $\mathrm{AB}_{2}\left(\mathrm{Zr}_{0,9} \mathrm{Ti}_{0,1} \mathrm{Ni}_{1,04} \mathrm{Mn}_{0,64} \mathrm{~V}_{0,46}\right)$

\begin{tabular}{|c|c|c|c|c|c|c|c|c|}
\hline \multirow{3}{*}{$\begin{array}{l}\text { Ligas } \\
\text { Porcentagens } \\
\% \mathrm{Zr}\end{array}$} & \multicolumn{2}{|c|}{$\mathrm{AB}_{2}$ sem tratamento } & \multicolumn{2}{|c|}{$\begin{array}{l}\mathrm{AB}_{2} \text { sem aditivos, } \\
\text { tratada por moagem }\end{array}$} & \multicolumn{2}{|c|}{$\begin{array}{c}\mathrm{AB}_{2} \text { tratada por } \\
\text { moagem com } 10 \% \text { de } \\
\mathrm{LaNi}_{4,7} \mathrm{Sn}_{0,3}\end{array}$} & \multicolumn{2}{|c|}{$\begin{array}{c}\mathrm{AB}_{2} \text { tratada por } \\
\text { moagem com } 10 \% \\
\text { de pó de } \mathrm{Ni}\end{array}$} \\
\hline & teórica & experim. & teórica & experim. & teórica & experim. & teórica & experim. \\
\hline & 28,1 & 25,8 & 28,1 & 25,8 & 25,2 & 22,6 & 25,2 & 22,7 \\
\hline$\% \mathrm{Ti}$ & 3,1 & 3,3 & 3,1 & 2,6 & 2,8 & 2,7 & 2,8 & 2,6 \\
\hline$\% \mathrm{Ni}$ & 34,4 & 33,5 & 34,4 & 33,7 & 38,8 & 33,8 & 38,8 & 38,5 \\
\hline$\% \mathrm{Mn}$ & 20,1 & 22,1 & 20,1 & 19,6 & 18,1 & 20,5 & 18,1 & 19,6 \\
\hline$\% \mathrm{~V}$ & 14,3 & 15,3 & 14,3 & 15,0 & 12,9 & 12,9 & 12,9 & 12,9 \\
\hline$\% \mathrm{La}$ & - & - & - & - & 1,7 & 1,4 & - & - \\
\hline$\% \mathrm{Sn}$ & - & - & - & - & 0,5 & 0,3 & - & - \\
\hline$\% \mathrm{Cr}^{*}$ & - & - & - & 0,5 & - & 0,5 & - & 0,8 \\
\hline$\% \mathrm{Fe}^{*}$ & - & - & - & 2,7 & - & 2,3 & - & 2,8 \\
\hline
\end{tabular}

* contaminantes 


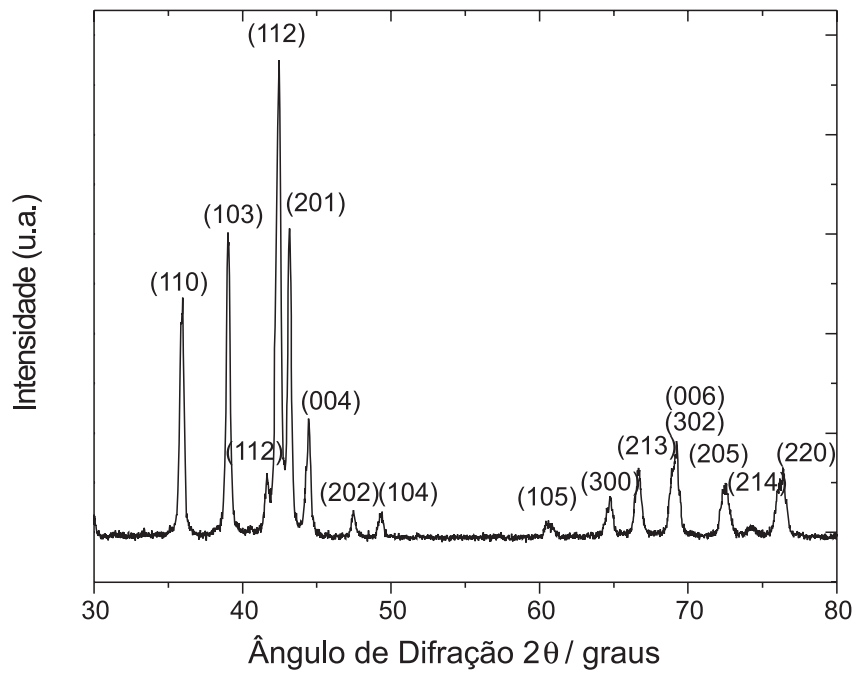

Figura 1. Difratograma de raios $X$ obtido para a liga $\mathrm{AB}_{2}$ $\left(\mathrm{Zr}_{0,9} \mathrm{Ti}_{0,1} \mathrm{Ni}_{1,04} \mathrm{Mn}_{0,64} \mathrm{~V}_{0,46}\right)$. Os números colocados acima de cada pico indicam os correspondentes índices de Miller referentes aos planos (hkl) onde ocorre a difração dos raios $X$

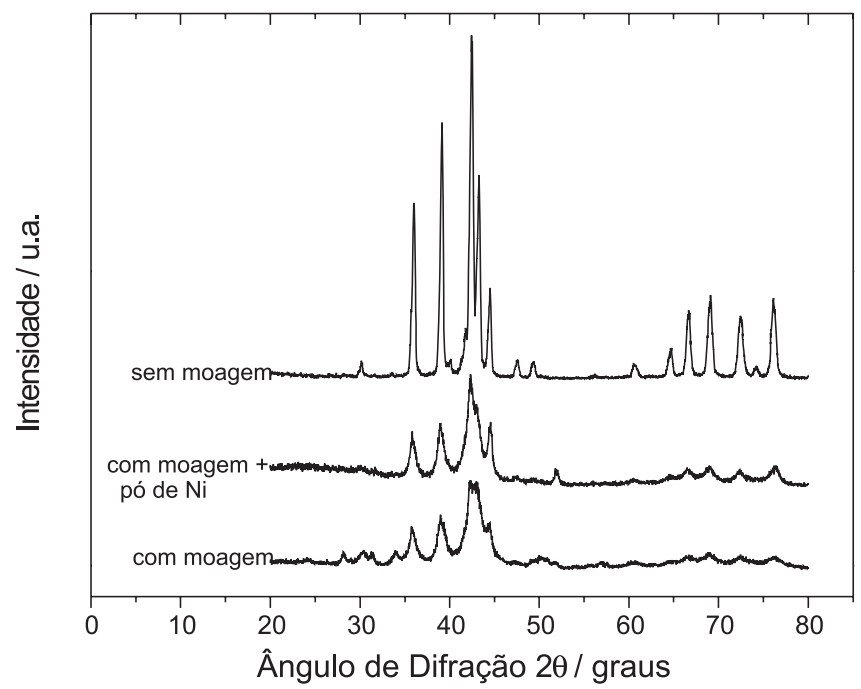

Figura 2. Difratogramas de raios $X$ obtidos para a liga $A B_{2}$ $\left(\mathrm{Zr}_{0,9} \mathrm{Ti}_{0,1} \mathrm{Ni} i_{1,04} \mathrm{Mn}_{0,64} \mathrm{~V}_{0,46}\right)$ sem tratamento por moagem, tratada por moagem sem aditivos e tratada por moagem com $10 \%$ de pó de $\mathrm{Ni}$

cristalitos, já que não foram observados deslocamentos dos picos para ângulos de difração maiores ou menores, fato que denotaria variações nos parâmetros de rede e, conseqüentemente, do volume da cela unitária. $\mathrm{O}$ volume da cela unitária é um dos fatores importantes relacionados com a estabilidade das ligas na fase hidreto.

O volume da célula unitária determina a magnitude do processo de expansão/contração da cela unitária decorrente da intercalação/desintercalação de hidrogênio, que ocorre durante os ciclos de carga/descarga. Este fenômeno leva à quebra das partículas, aumentado a área de contato com o ambiente corrosivo (eletrólito alcalino) e acentuando os processos de corrosão pelo eletrólito. Um maior valor de volume da cela unitária indica um maior valor dos espaços intersticiais que acomodam o hidrogênio na fase hidreto. Neste caso, a formação da fase hidreto deve resultar em uma menor compressão do hidrogênio absorvido que acaba diminuindo a expansão da cela cristalina unitária e a ruptura das partículas da liga, levando a uma maior estabilidade do eletrodo ${ }^{20}$. No presente estudo, a modificação das ligas por moagem com Ni e $\mathrm{LaNi}_{4,7} \mathrm{Sn}_{0,3}$ não levou a mudanças mensuráveis no volume da cela unitária, indicando um efeito desprezível sobre a estabilidade à ciclagem.

Um aspecto importante mencionado anteriormente é a pequena contaminação das amostras com $\mathrm{Fe}$ e $\mathrm{Cr}$ que ocorre durante a moagem, conforme revelado pela microanálise por EDX. Pelos resultados de DRX observou-se que os picos principais indicativos da presença destes elementos deveriam aparecer em torno de $2 \theta=$ $44,5^{\circ}$, superpondo-se ao sinal do plano (004) da liga; entretanto, nenhuma evidência da presença deste pico ou de superposição é notada nesta região, fato que provavelmente decorre do baixo teor destes contaminantes. A presença dos contaminantes também não levou a alterações significativas no posicionamento dos picos de difração de raios $X$ em relação à liga base, fato que denota um efeito desprezível sobre o volume de cela unitária e, portanto, sobre os processos de pulverização e de estabilidade do material ativo.

A partir de medidas de potencial em função do tempo ao longo dos ciclos de descarga galvanostáticos é possível traçar os perfis de descarga para as ligas estudadas, que correspondem aos gráficos do potencial do eletrodo em função do tempo de descarga. Na Figura 3 são apresentados estes resultados para vários ciclos entre o $1^{\circ} \mathrm{e} \mathrm{o}$ $30^{\circ}$, obtidos utilizando-se uma densidade de corrente de $100 \mathrm{~mA} \mathrm{~g}^{-1}$, para o eletrodo formado pela liga $\mathrm{Zr}_{0,9} \mathrm{Ti}_{0,1} \mathrm{Ni}_{1,04} \mathrm{Mn}_{0,64} \mathrm{~V}_{0,46}$ sem tratamento por moagem. Analisando-se estes resultados observa-se que a cada ciclo o patamar de potencial do eletrodo se torna mais negativo e que o tempo máximo de descarga avança para maiores valores. Estes dois comportamentos são causados por mudanças na cinética de absorção/dessorção de hidrogênio com o aumento do número de ciclos. Tal fato tem sido explicado levando-se em conta que a cinética de hidretação da liga aumenta durante os primeiros ciclos devido às transformações que ocorrem na superfície das partículas causadas, principalmente, pela redução e ruptura de uma camada de óxidos inicialmente presente.

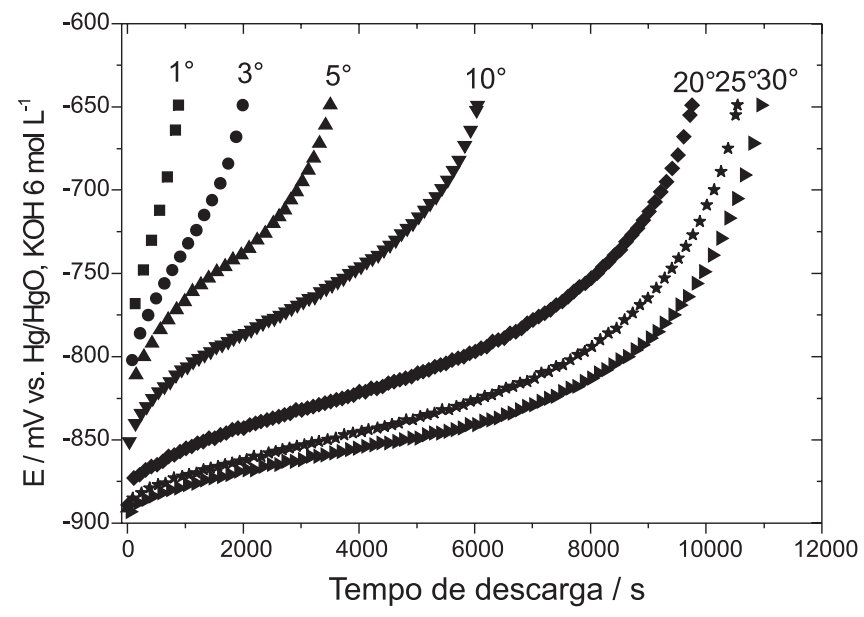

Figura 3. Perfil de descarga para o eletrodo formado pela liga $\mathrm{Zr}_{0,9} \mathrm{Ti}_{0,1} \mathrm{Ni} i_{1,04} \mathrm{Mn} n_{0,64} V_{0,46}$ entre o $1^{\circ}$ e o $30^{\circ}$ ciclo

A Figura 4 mostra os perfis de capacidade de descarga em função do número de ciclos de carga/descarga para os eletrodos formados pela liga $\mathrm{AB}_{2}$ com e sem os tratamentos superficiais. Nesta Figura os valores da capacidade de descarga foram normalizados e apresentados como $\mathrm{Q} / \mathrm{Q}_{\text {máx. }}$, sendo $\mathrm{Q}$ a capacidade real da liga $\mathrm{e}$ 


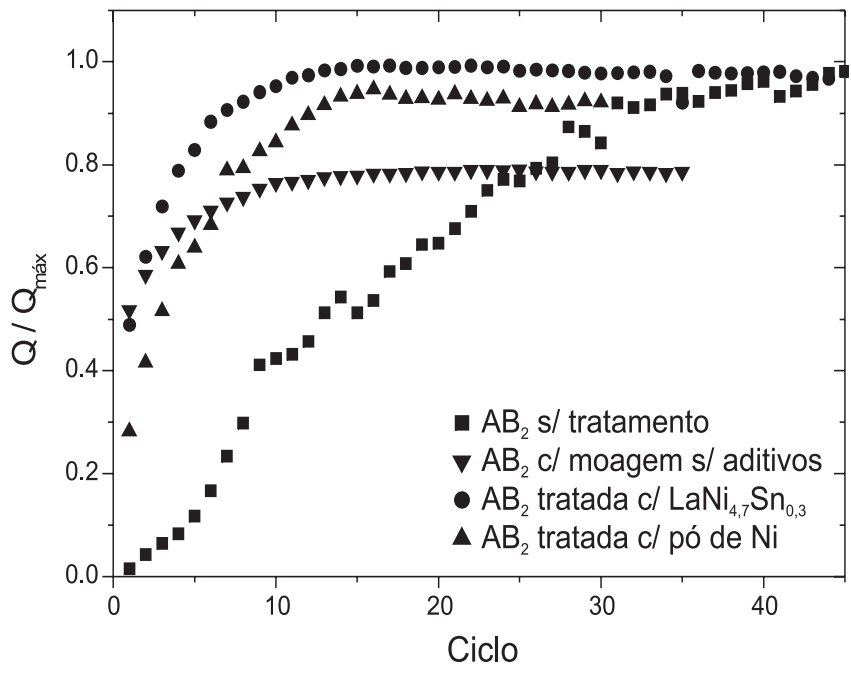

Figura 4. Capacidade de descarga do eletrodo formado pelas ligas $A B_{2}$ não tratada e tratadas com $\mathrm{LaNi}_{4,7} \mathrm{Sn}_{0,3}$ e $\mathrm{Ni}$

$\mathrm{Q}_{\text {máx. }}$ a capacidade máxima obtida para a liga não tratada (320 mAh $\mathrm{g}^{-1}$ ). $\mathrm{O}$ valor de $\mathrm{Q}$ é obtido a partir do produto da corrente pelo tempo máximo de descarga (Figura 3), normalizado pela massa da liga presente no eletrodo.

Observa-se que a capacidade de armazenamento de carga da liga $\mathrm{AB}_{2}$ sem tratamento aumenta muito lentamente com o decorrer do número de ciclos, atingindo um patamar $\left(\mathrm{Q}_{\text {máx. }} \sim 320 \mathrm{mAh} \mathrm{g}^{-1}\right)$ após 35 ciclos de carga e descarga. No entanto, para a liga $A_{2}$ sem os aditivos mas submetida à moagem ocorre uma diminuição do tempo de ativação, sendo acompanhada por uma grande diminuição do Q máximo. Estes fatos podem ser atribuídos à maior pulverização do material que deve ser acompanhada por uma forte oxidação da liga durante o tratamento por moagem. Para o eletrodo formado com essa mesma liga submetida ao tratamento superficial com $10 \%$ de $\mathrm{LaNi}_{47} \mathrm{Sn}_{03}$ também se verifica uma ativação mais rápida ( 12 ciclos), mas neste caso não há diminuição do Q máximo. O mesmo comportamento em relação ao tempo de ativação é observado para a liga tratada com $10 \%$ de pó de Ni. No entanto, verifica-se uma pequena diminuição do Q máximo, que é explicada pelo fato de que o $\mathrm{Ni}$ incorporado à liga não participa do processo de armazenamento de carga. Na literatura ${ }^{21}$, a diminuição do tempo de ativação de outras ligas baseadas em zircônio tratadas com $\mathrm{Ni}$ é explicada com base no fato de que a superfície rica em níquel não apenas diminui o acúmulo de Zr na superfície das partículas, mas também catalisa a reação eletródica. No presente trabalho, a diminuição do tempo de ativação da liga base após o tratamento com $\mathrm{LaNi}_{4,7} \mathrm{Sn}_{0,3}$ pode ser atribuída ao fato de que esta liga possui uma boa atividade eletroquímica inicial e, como está aderida à superfície da liga base, atua como sítio ativo acelerando a reação eletroquímica. Assim, durante os ciclos de carga e descarga, os átomos de $\mathrm{H}$ são gerados e incorporados nos sítios superficiais contendo $\mathrm{LaNi}_{4,7} \mathrm{Sn}_{0,3}$, difundindo-se em seguida para o interior das partículas da liga base.

A pressão de equilíbrio de hidrogênio nas ligas de hidreto metálico foi obtida mediante medidas do potencial de equilíbrio do eletrodo, com a utilização da Equação de Nernst ${ }^{16,17}$, sendo este parâmetro relacionado com o processo de auto-descarga do eletrodo. A Figura 5 mostra estes resultados para as ligas não tratada e tratadas por moagem, em função do estado de carga. Em todos os casos, nota-se uma diminuição contínua da pressão de equilíbrio de hidrogênio à medida em que se diminui o estado de carga do eletrodo. Estes resultados sugerem que para uma bateria selada, um máximo de pressão interna de hidrogênio (cerca de $3 \mathrm{~atm}$ ) será atingido quando o eletrodo se encontrar $100 \%$ carregado. Não há um platô definido da pressão de equilíbrio de hidrogênio para todas as ligas estudadas, o que evidencia ausência de transformações de fases ao longo do processo de descarga, como normalmente verifica no caso de ligas $\mathrm{AB}_{5}{ }^{22}$. Dentro dos erros experimentais inerentes à técnica de medida e à reprodutibilidade de preparação dos materiais e eletrodos, os resultados evidenciam um efeito marginal sobre a pressão de equilíbrio de hidrogênio introduzido pelos processos de moagem aqui considerados. Isto indica que não deve ter havido variações apreciáveis da estrutura cristalina dos materiais (eg. amorfização) após estes tratamentos, sendo isto confirmado pelos resultados de difração de raios $\mathrm{X}$.

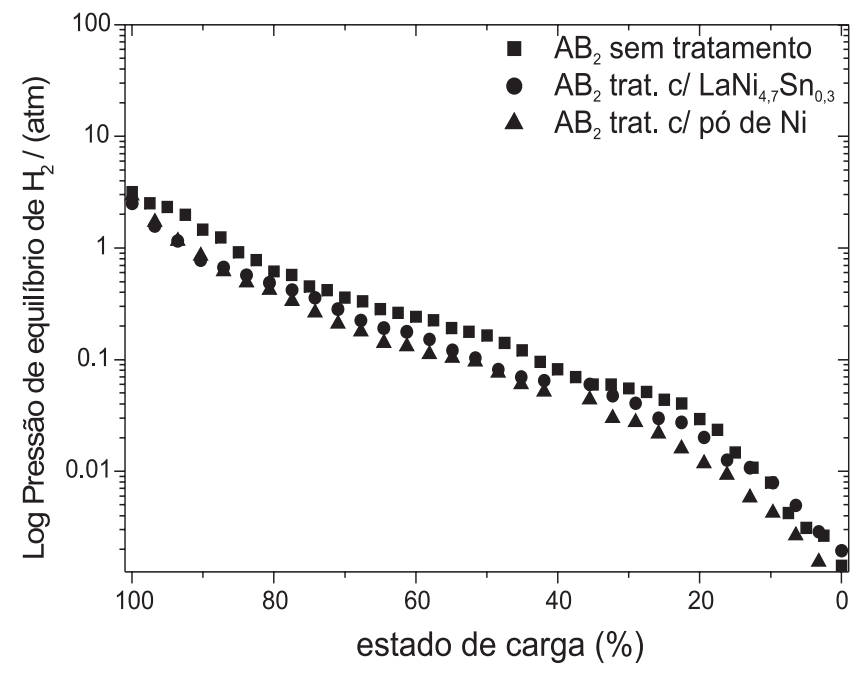

Figura 5. Pressão de equilíbrio de hidrogênio em função do estado de carga dos eletrodos com as ligas $A B_{2}$ não tratadas e tratadas por moagem

As Figuras 6 e 7 apresentam os diagramas de impedância Nyquist obtidos em diferentes estados de carga e diversas temperaturas, ilustrando o comportamento em baixas e altas freqüências, para o eletrodo preparado com a liga $\mathrm{AB}_{2}$ tratada com $10 \%$ de $\mathrm{LaNi}_{4,7} \mathrm{Sn}_{0,3}$. Observa-se claramente o aparecimento de dois arcos, um em altas e outro em baixas freqüências. No caso da liga descarregada ( $0 \%$ de estado de carga), há uma tendência de crescimento da impedância no limite de baixas frequências. Este comportamento foi qualitativamente o mesmo para todas as ligas estudadas neste trabalho.

O primeiro semi-círculo, observado no domínio de altas freqüências (Figuras 6b e 7b), (10 kHz a $100 \mathrm{~Hz})$ tem sido freqüentemente associado à resistência de contato entre o coletor de corrente (tela de níquel), o pó de carbono e a liga metálica, em particular para ligas do tipo $\mathrm{AB}_{5}{ }^{21}$. No entanto, há uma diferença fundamental do comportamento dos diagramas de impedância nesta região, para os sistemas estudados neste trabalho, em relação aos resultados para a liga $\mathrm{AB}_{5}{ }^{22}$. Na Figura $7 \mathrm{~b}$ verifica-se que os arcos em altas freqüências denotam uma dependência exponencial da velocidade do processo cinético associado em função da temperatura (ver Figura 8), fato que não ocorre para a liga $\mathrm{AB}_{5}^{23}$, onde a magnitude do arco permanece aproximadamente constante. O outro semicírculo na região de frequiências intermediárias $(10 \mathrm{~Hz}$ a $1 \mathrm{mHz})$ é normalmente atribuído às relaxações relativas à etapa de transferência de carga, que se verifica na interface metal/solução. Finalmente, para os casos em que o estado de carga tende a zero, o 

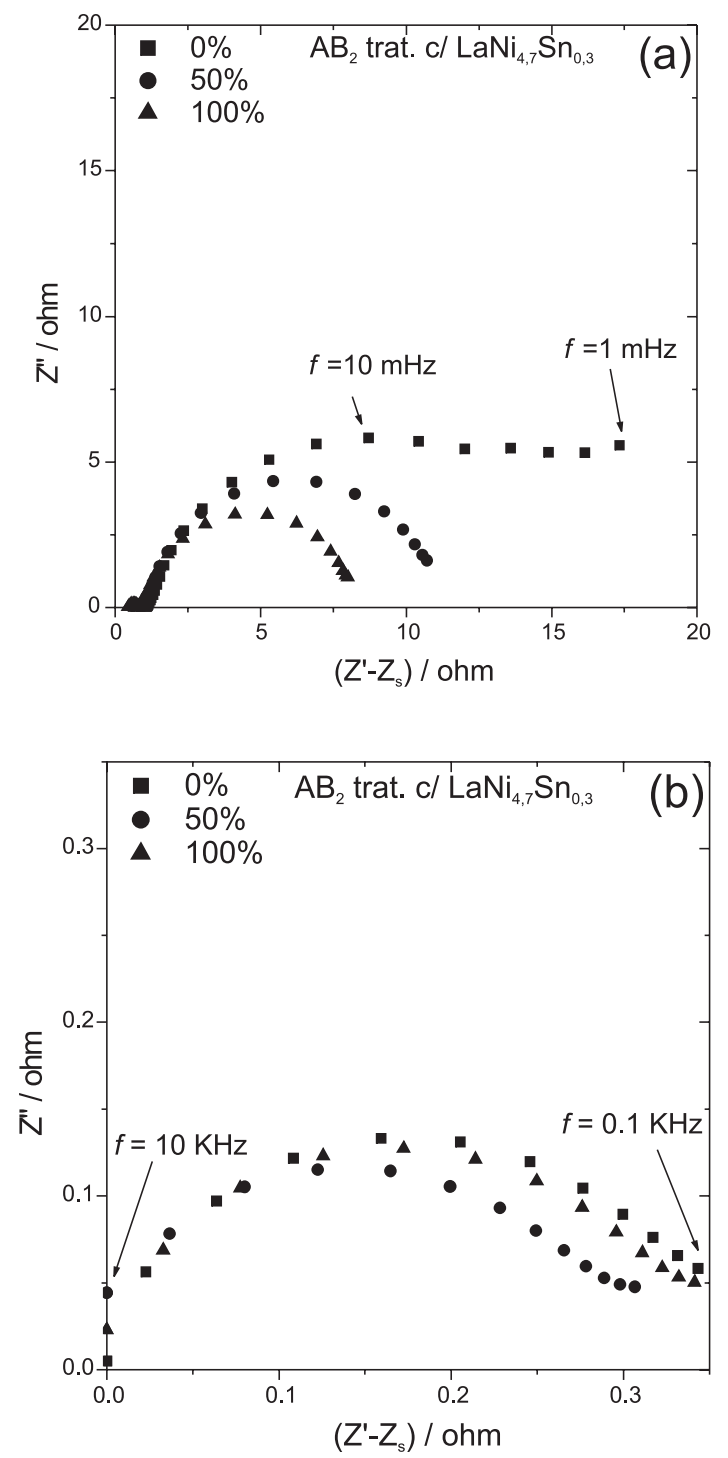

Figura 6. Diagramas de Nyquist obtidos para o eletrodo preparado com o pó da liga $\mathrm{Zr}_{0,9} \mathrm{Ti}_{0,1} \mathrm{Ni}_{1,04} \mathrm{Mn}_{0,64} \mathrm{~V}_{0,40}$ tratada com $\mathrm{LaNi} \mathrm{i}_{4,5} \mathrm{Sn}_{0,3}$, mostrando o efeito do estado de carga: (a) baixas freqüências e (b) altas freqüências. $T=26^{\circ} \mathrm{C}$, onde Z' e Z" representam os componentes real e imaginário da impedância

aumento acentuado do componente imaginário da impedância no domínio de baixas frequiências tem sido atribuído a fenômenos de relaxação vinculados ao transporte difusional de hidrogênio atômico no interior das partículas da liga. As Figuras 6a e 7a evidenciam que os comportamentos dos arcos em frequiências médias e pequenas são similares aos descritos na literatura. A única diferença relaciona-se ao comportamento do arco em média frequiência em função do estado de carga. Enquanto que na Figura 6(a) a magnitude do arco decresce com o aumento do estado de carga, no caso das ligas $\mathrm{AB}_{5}$ o comportamento é oposto ${ }^{24}$.

A dependência exponencial da magnitude do arco de alta freqüência com a temperatura (Figura 8) indica que o correspondente processo é controlado por ativação, o que descarta a possibilidade de ser relacionado com problemas resistivos de contato elétrico, mas não exclui a possibilidade de que se refira a um processo resistivo associado à presença deficitária de eletrólito em um filme poroso de óxido/hidróxido que deve recobrir as partículas da liga. Por outro lado, publicações anteriores ${ }^{23-25}$ sobre sistemas $\mathrm{AB}_{2}$ e $\mathrm{AB}_{5}$ têm sugerido que o arco em menor frequiência deve ser relativo à
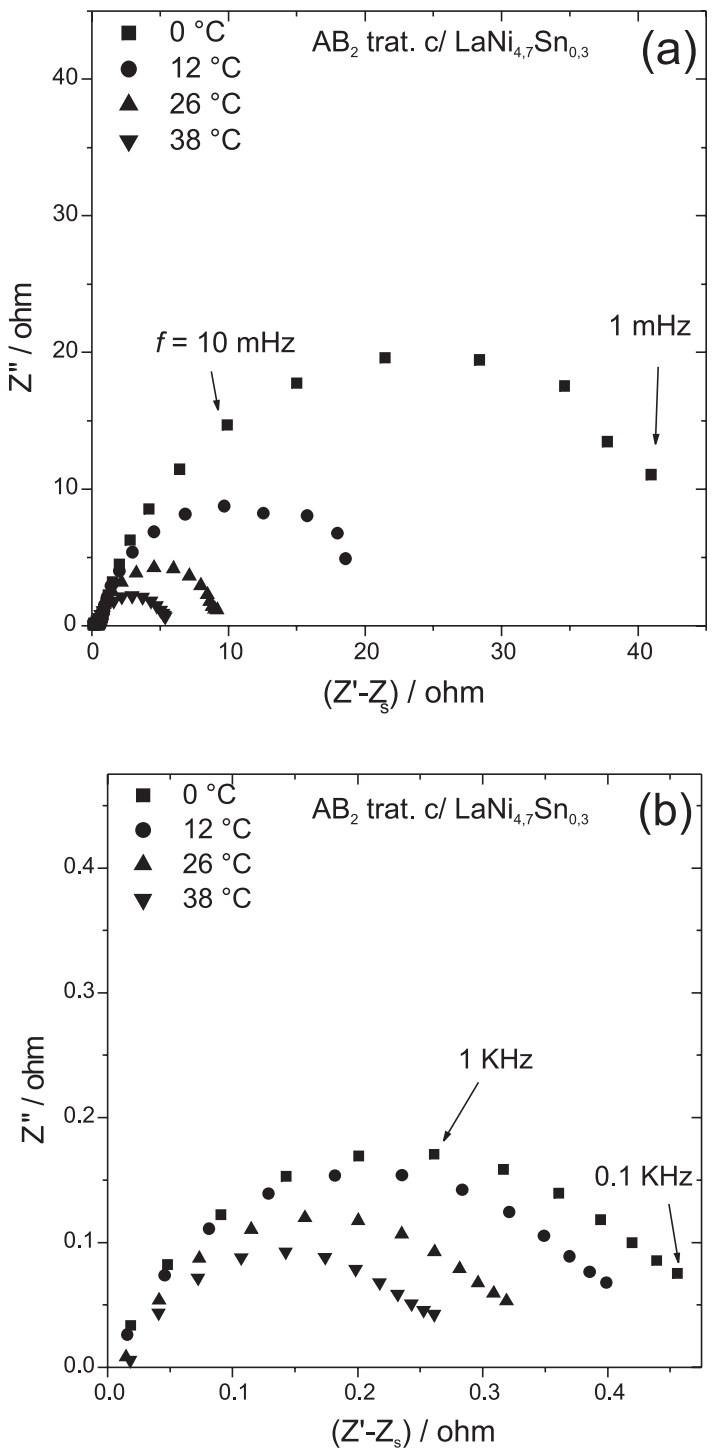

Figura 7. Diagramas de Nyquist obtidos para o eletrodo preparado com o pó da liga $\mathrm{Zr}_{0,9} \mathrm{Ti}_{0,1} \mathrm{Ni}_{1,04} \mathrm{Mn}_{0,64} \mathrm{~V}_{0,46}$, tratada com $\mathrm{LaNi}_{4,7} \mathrm{Sn}_{0,3}$ (estado de carga $=100 \%$ ), mostrando o efeito da temperatura: (a) baixas frequiências e (b) altas freqüências

etapa de transferência de carga, que se verifica na interface metal/ solução, em conexão paralela com a capacitância da dupla camada elétrica ${ }^{25}$. Para ampliar a abordagem da presente análise, os valores do recíproco das resistências de polarização associados a ambos os arcos foram graficados em função do recíproco da temperatura, para se obter os valores das energias de ativação dos correspondentes processos, utilizando-se a equação de Arrhenius. As Figuras 8 e 9 apresentam os gráficos de Arrhenius, que demonstram a relação linear entre o logaritmo natural dos valores de $\mathrm{R}_{\mathrm{p}}^{-1}$ e o recíproco da temperatura na escala termodinâmica. A Tabela 2 apresenta os valores correspondentes das energias de ativação obtidos para todas as ligas no estado $100 \%$ carregadas.

Analisando os dados das Figuras 8 e 9 a uma dada temperatura, nota-se que ambos os conjuntos de valores de $\mathrm{R}_{\mathrm{p}}^{-1}$ variam para as diferentes ligas, porém, em todos os casos, diminuem com o aumento da temperatura. Devido ao fato de que os tamanhos das partículas são desconhecidos para as ligas no estado ativado torna-se imprópria qualquer tentativa de associar os valores de $\mathrm{R}_{\mathrm{p}}^{-1}$ obtidos para as diferentes ligas com as propriedades catalíticas específicas 


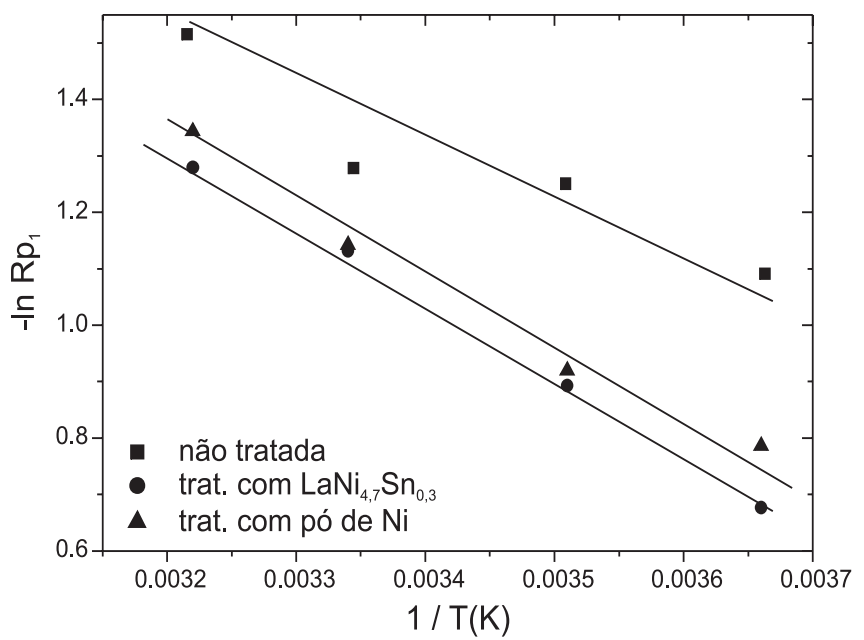

Figura 8. Relação linear entre o logaritmo natural da resistência de polarização em altas freqüências e o inverso da temperatura, em Kelvin, para as diversas ligas estudadas

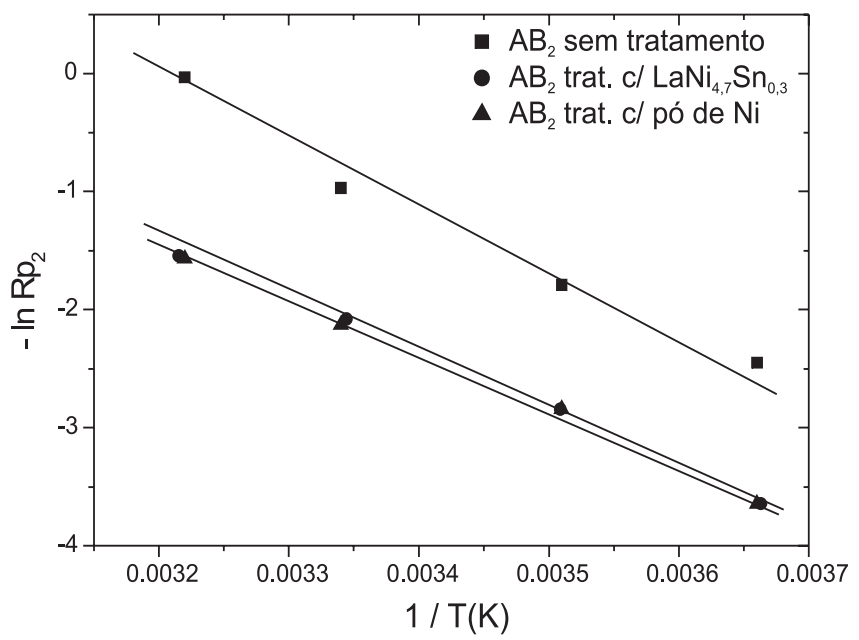

Figura 9. Relação linear entre o logaritmo natural da resistência de polarização em médias freqüências e o inverso da temperatura, em Kelvin, para as diversas ligas estudadas

Tabela 2. Valores da energia de ativação $\mathrm{Ea}^{\neq}$obtidos para as ligas $\mathrm{AB}_{2}$ não tratadas e tratada

\begin{tabular}{|c|c|c|}
\hline Liga & $\mathrm{E}_{\mathrm{a} 1} \neq\left(\mathrm{kJ} \mathrm{mol}^{-1}\right)$ (alta freqüência) & $\mathrm{E}_{\mathrm{a} 2}^{\neq}\left(\mathrm{kJ} \mathrm{mol}^{-1}\right)$ (média freqüência) \\
\hline $\mathrm{AB}_{2}$ não tratada & 9,5 & 46 \\
\hline $\mathrm{AB}_{2}$ tratada com $\mathrm{LaNi}_{4,7} \mathrm{Sn}_{0,3}$ & 10,4 & 44 \\
\hline $\mathrm{AB}_{2}^{2}$ tratada com pó de $\mathrm{Ni}$ & 10,4 & 42 \\
\hline
\end{tabular}

das mesmas. No entanto, uma comparação direta do desempenho cinético dos sistemas pode ser feita através dos correspondentes valores de $\mathrm{E}_{\mathrm{a}}^{\neq}$, já que este parâmetro, em princípio, não depende do tamanho de partículas. Este termo somente depende do material eletródico, sendo, conforme mencionado anteriormente, o valor relativo à alta freqüência $\left(\mathrm{E}_{\mathrm{a} 1}{ }^{*}\right)$ mais provavelmente relacionado com um efeito resistivo associado à migração do eletrólito contido em um filme poroso de óxido/hidróxido presente na superfície da liga e aquele em médias freqüências referente ao processo de transferência de carga do processo de hidretação.

Os valores $\mathrm{E}_{\mathrm{a} 1}{ }^{\neq}$e $\mathrm{E}_{\mathrm{a} 2}{ }^{\neq}$dados na Tabela 2 indicam que o tratamento por moagem não leva a mudanças significativas nas cinéticas do processo migratório do eletrólito ou da reação de transferência de carga nos diferentes materiais. Assim, conclui-se que a melhora mais significativa na cinética do processo eletroquímico global introduzida pela cobertura da liga $\mathrm{AB}_{2}$ com Ni ou $\mathrm{LaNi}_{47} \mathrm{Sn}_{03}$ restringe-se apenas aos ciclos iniciais de carga/descarga, acelerando o processo de ativação, apenas até que a liga atinja sua capacidade máxima.

\section{CONCLUSÕES}

Pelo difratograma de raios $X$ do pó da liga $A_{2}$ $\left(\mathrm{Zr}_{0,9} \mathrm{Ti}_{0,1} \mathrm{Ni}_{1,04} \mathrm{Mn}_{0,64} \mathrm{~V}_{0,46}\right)$ não tratada foi observada a presença de uma fase hexagonal pura do tipo $\mathrm{C} 14$ (estrutura tipo-MgZn $\mathrm{n}_{2}$ ). As curvas de carga/descarga mostraram que a capacidade da liga $\mathrm{AB}_{2}$ sem tratamento aumenta muito lentamente com o decorrer do número de ciclos de carga/descarga, enquanto que os eletrodos formados com essa mesma liga $\mathrm{AB}_{2}$ porém submetida aos tratamentos superficiais com $10 \%$ de $\mathrm{Ni}$ e $\mathrm{LaNi}_{4,7} \mathrm{Sn}_{0,3}$ apresentam ativação muito mais rápida. Os resultados de pressão de equilíbrio de hidrogênio mostraram que não há um platô definido da pressão de hidrogênio para todas as ligas estudadas, observando-se apenas uma diminuição marginal dos valores da pressão causada por ambos os tratamentos superficiais.

Os valores da resistência de polarização obtidos através de medidas de impedância eletroquímica permitiram estimar os valores da energia de ativação da reação de transferência de carga envolvida na etapa de hidretação/desidretação, tendo-se observado alta similaridade da cinética reacional nas várias amostras analisadas.

\section{AGRADECIMENTOS}

À Fundação de Amparo à Pesquisa do Estado de São Paulo, (FAPESP) pelos auxílios financeiros e bolsas concedidas e Conselho Nacional de Desenvolvimento Científico e Tecnológico (CNPq).

\section{REFERÊNCIAS}

1. Lee, H. Y.; Goo, N. H.; Jeong, W. T.; Lee, K. S.; J. Alloys Compd. 2000, $313,258$.

2. Chen, W. X.; Xu, Z. D.; Tu, J. P.; Int. J. Hydrogen Energy 2002, 27, 439.

3. Orimo, S.; Zuttel, A.; Ikeda, K.; Saruki, S.; Fukunaga, T.; Fujii, H.; Schlapbach, L.; J. Alloys Compd. 1999, 293-295, 437.

4. Ratnakumar, B. V.; Witham, C.; Bowman, Jr. R. C.; Hightower, A.; Fultz, B.; J. Electrochem. Soc. 1996, 143, 2578.

5. Reilly, J. J.; Adzic, G. D.; Johnson, J. R.; Vogt, T.; Mukerjee, S.; McBreen, J.; J. Alloys Compd. 1999, 293-295, 569.

6. Ambrosio, R. C.; Ticianelli, E. A.; Quim. Nova 2001, 24, 245.

7. Liu, B. H.; Li, Z. P.; Okutsu, A.; Suda, S.; J. Alloys Compd. 2000, 296, 148.

8. Xu, Y.; Chen, C.; Wang, X.; Lei, Y.; Wang, Q.; J. Alloys Compd. 2002, 337, 214.

9. Huot, J.; Liang, G.; Boily, S.; Neste, A. V.; Schulz, R.; J. Alloys Compd. 1999, 293-295, 495.

10. Iwakura, C.; Norhara, S.; Inoue, H.; Fukumoto, Y.; Chem. Commun. 1996, $15,1831$.

11. Zaluska, A.; Zaluski, L.; Strom-Olsen, J. O.; J. Alloys Compd. 1999, 289, 197. 
12. Yang, Q. M.; Ciureoni, M.; Ryan, D. H.; Ström, O.; J. Alloys Compd. 1998 , 274, 266.

13. Yang, K.; Chen, D.; Chen, L.; Zhang, H.; Sun, W.; Li, Y.; J. Alloys Compd. 1999, 293-295, 670.

14. Sitaram, V.; Mani, N.; Kesavan, T. R.; Ramaprabhu, S.; Int. J. Hydrogen Energy 2002, 27, 413 .

15. Cui, N.; He, P.; Luo, J. L.; Electrochim. Acta 1999, 44, 3549.

16. Notten, P. H.; Latroche, M.; Percheron-Guégan, A.; J. Electrochem. Soc. 1999, 146, 3181.

17. Liang, G.; Huot, J.; Schulz, R.; J. Alloys Compd. 2001, 320, 133.

18. Santos, A. R.; Ambrosio, R. C.; Ticianelli, E. A.; Int. J. Hydrogen Energy 2004, 29, 1253
19. Triaca, W. E.; Peretti, H. A.; Corso, H. L.; Bonesi, A.; Visintin, A.; J. Power Sources 2003, 113, 151.

20. Iwakura, C.; Inoue, H.; Nohara, S.; Shin-Ya, R.; Kurosaka, S.; Miyanohara, K.; J. Alloys Compd. 2002, 330-332, 636.

21. Wang, C.; J. Electrochem. Soc. 1998, 145, 1801.

22. Chen, Z.; Chen, Z.; Huang, K.; Huang, P.; J. Alloys Compd. 1999, $293-$ 295,712 .

23. Ticianelli, E. A.; Mukerjee, S.; McBreen, J.; Adzic, G. D.; Johnson, J. R.; Reilly, J. J.; J. Electrochem. Soc. 1999, 146, 3582.

24. Souza, E. C.; Ticianelli, E. A.; J. Braz. Chem. Soc. 2003, 14, 544.

25. Castro, E. B.; Real, S. G.; Bonesi, A.; Visintin, A.; Triaca, E.; Electrochim. Acta 2004, 49, 3879. 